\title{
Rare Occurrence of the JAK1 Mutation in Acute Promyelocytic Leukemia Patients
}

\author{
Jia Yin Yang Xu Jinlan Pan Chunxiao Wu Qinrong Wang Hong Yao \\ Lijun Wen Xiaopeng Tian Depei Wu Aining Sun Suning Chen
}

The First Affiliated Hospital of Soochow University, Jiangsu Institute of Hematology, Key Laboratory of Thrombosis and Hemostasis of Ministry of Health, Suzhou, PR China

Acute promyelocytic leukemia (APL) is a distinct subtype of acute myeloid leukemia characterized by a chromosome translocation of $\mathrm{t}(15 ; 17)(\mathrm{q} 24 ; \mathrm{q} 21)$ which involves fusion of the retinoic acid receptor $\alpha(R A R \alpha)$ gene in chromosome 17 and the promyelocytic leukemia (PML) gene in chromosome 15. The fusion protein encoded by the $P M L /$ $R A R \alpha$ gene makes APL sensitive to all-trans retinoic acid, a derivative of vitamin $\mathrm{A}$. With the introduction of all-trans retinoic acid and arsenic trioxide in the past several decades, the complete remission (CR) rate and 5-year diseasefree survival for APL were elevated to more than 90\% [1].

The Janus kinase (JAK) is a nonreceptor tyrosine kinase, including 4 family members, i.e. JAK1, JAK2, JAK3, and TYK2. The JAK1 gene is organized into 25 exons, including 24 coding exons, and spans about $133.28 \mathrm{~Kb}$ in the chromosome $1 \mathrm{p} 31.3$ region, which is responsible for transducing cytokine-induced signals via JAK-STAT pathway aberrations; JAK1 signal activation is partially responsible for leukemogenesis. Recently, Wartman et al. [2] found Jak1 (V657F) in a murine APL model via whole genomic sequencing. This Jak1 (V657F) was identified in 6 other mice that were not closely related to the proband [2]. After analyzing 186 acute adulthood leukemias, 30 multiple myelomas, and 278 common solid cancers, Jeong et al. [3] discovered $4 J A K 1$ mutations in patients with acute leukemia. Among them, the JAK1 mutations

\section{KARGER}

E-Mail karger@karger.com

www.karger.com/aha
V658F and L783F occurred in 4 leukemia patients, while the p.V658F mutation was found in 1 patient with APL [3]. In our previous report, the JAK1 mutation was found in 3 out of 49 in acute T-lymphocytic leukemia patients [4]. To explore the frequency of the JAK1 mutation in APL, we performed $J A K 1$ gene sequencing in a group of APL patients in our center. This study was approved by the Ethics Committee of the First Affiliated Hospital of Soochow University. Informed consent was provided following the Declaration of Helsinki.

A total of 86 patients with de novo APL were screened in all 24 exons of JAK1 using genomic DNA-PCR amplification sequencing. The clinical characteristics of these patients are listed in table 1 . Bone marrow was aspirated and cultured with colcemid overnight at $37^{\circ} \mathrm{C}$ and harvested according to standard procedures [5]. Karyotypic analysis was performed on R-banded metaphases and described according to the recommendations of the ISCN. Metaphases were analyzed by $\mathrm{R}$ banding and karyotype showed that 82 out of 84 patients were $t(15 ; 17)$ with a positive $P M L / R A R \alpha$ fusion gene, while 1 patient displayed $\mathrm{t}(11 ; 17)$ with the PLZF/RAR $\alpha$ fusion gene. Total DNA was extracted from bone marrow using a DNA

J. Yin and Y. Xu contributed equally to this work.
C 2013 S. Karger AG, Basel

0001-5792/13/1304-0251\$38.00/0
Aining Sun and Suning Chen

The First Affiliated Hospital of Soochow University, Jiangsu Institute of Hematology Key Laboratory of Thrombosis and Hemostasis of Ministry of Health Suzhou, Jiangsu Province 215006 (PR China)

E-Mail ainingsun@ hotmail.com and chensuning@sina.com 


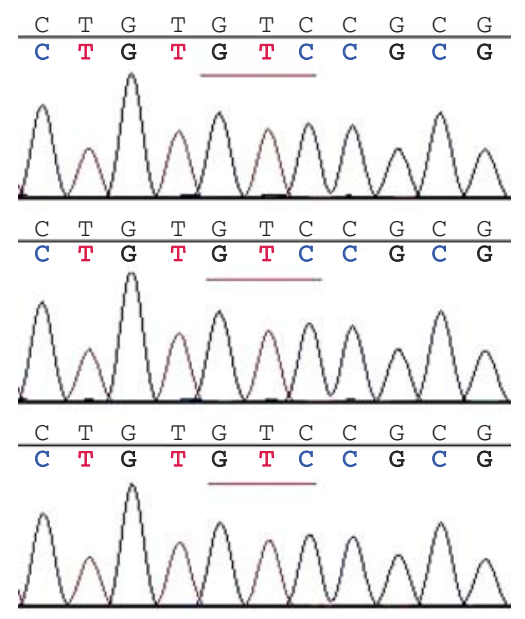

Table 1. Clinical characteristic of patients with APL

\section{Characteristics}

Patients, $\mathrm{n}$

Age, years

Male/female ratio

White blood cells, $\times 10^{9} / 1$

Hemoglobin, g/l

Platelets, $\times 10^{9} / 1$

Bone marrow blasts, $\%$

Cytogenetics

$\mathrm{t}(15 ; 17)$ with PML/RARa

$\mathrm{t}(11 ; 17)$ with PLZF/RARa

85

$34(7-79)$

$45 / 40$

$7.50(0.95-194.7)$

$94(21-132)$

$24.5(3-145)$

$87.7(56-98.4)$

84

1

Values are presented as medians (range) unless otherwise stated.

Fig. 1. Sequencing diagram of a representative specimen in the V658F area where mutations have been reported by earlier studies. The coding sequence for residue V658 is underlined.

Table 2. Characteristics of APL patients with JAK1 new SNPs and silent mutation

\begin{tabular}{llllllll}
\hline Case & Sex & Age, years & Change(s) & Exon & Type of change & Protein level & Status \\
\hline 1 & female & 60 & c.277C $>$ T & 4 & SNP & Arg93Cys & novel \\
2 & male & 24 & c.677A $>$ G & 7 & SNP & Asn226Ser & novel \\
3 & male & 50 & c.655A $>$ G & 7 & SNP & Asn252Ser & Asn553Asn \\
4 & female & 16 & c.1659C $>$ T & 12 & synonymous & novel \\
\hline
\end{tabular}

Mini Kit (PureLink; Invitrogen, USA) according to the manufacturer's instructions. PCRs were performed in a volume of $25 \mu \mathrm{l}$ using 23 pairs of primers for all 24 JAK1 coding exons. The PCR products were separated on $2 \%$ agarose and visualized with GelRed. Direct sequencing was carried out with the Big Dye Terminator v3.1 Cycle Sequencing Reaction (Applied Biosystems) and was run on an ABI 3730xL DNA analyzer.

DNA sequencing analysis of the JAK1 gene found 4 novel heterozygous single-nucleotide variations which were not included in the NCBI SNP database. c.1659C $>$ T (Asn553Asn) was a synonymous mutation and the other three were nonsynonymous mutations. All four mutations were genomic variants rather than somatic mutations since they were identified in the CR samples (table 2).

The genetic variation of the $J A K 1$ gene in APL patients is unknown. By sequencing a large cohort of patients, we found 4 novel SNPs in patients with CR. Thus, our results have shown that JAK1 mutation is rare in newly diagnosed APL patients, suggesting that these mutations in JAK1 were unlikely to be involved in the pathogenesis of APL. Earlier studies identified the mutations in the same amino acid (or homologous amino acids) of V658F; however, we did not find these mutations in any of the 84 specimens (fig. 1). Because of the low frequency of the JAK1 mutation in APL, larger patient cohorts are needed for further study.

\section{Acknowledgements}

This work was supported by research grant BK2010206 from the Scientific Research Foundation of Jiangsu Province, research grant 20100145 from the Chinese Medical Doctor Association, and a project funded by the Priority Academic Program Development of Jiangsu Higher Education Institution (PAPD). 


\section{References}

JAK1 Mutation in APL Patients
1 Wang ZY, Chen Z: Acute promyelocytic leukemia: from highly fatal to highly curable. Blood 2008;111:2505-2515.

-2 Wartman LD, Larson DE, Xiang Z: Sequencing a mouse acute promyelocytic leukemia genome reveals genetic events relevant for disease progression. J Clin Invest 2011;121: 1445-1455.

-3 Jeong EG, Kim MS, Nam HK, Min CK, Lee S, Chung YJ, Yoo NJ, Lee SH: Somatic mutations of JAK 3 and JAK1 in acute leukemias and solid cancers. Clin Cancer Res 2008;14:3716-3721.
4 Wang Q, Qiu HY, Jiang H, Wu LL, Dong SS, Pan JL, Wang WJ, Ping NN, Xia J, Sun A, Wu DP, Xue YQ, Drexler HG, Macleod RA, Chen SN: Mutations of PHF6 are associated with mutations of NOTCH1, JAK1 and rearrangement of SET-NUP214 in T-cell acute lymphoblastic leukemia. Haematologica 2011;96: 1808-1814.

5 Saunders K, Czepulkowski B: Culture of human cells for chromosomal analysis; in Czepulkowski B (ed): Analyzing Chromosomes. Oxford, BIOS, 2001, pp 19-48. 